\title{
RESPUESTA DE Pinus pinceana GORDON A ESTRÉS POR SEQUÍA Y ALTAS TEMPERATURAS
}

\author{
RESPONSE OF Pinus pinceana Gordon TO DROUGHT AND HIGH TEMPERATURE STRESS
}

\author{
Rodrigo J. Martiñón-Martínez ${ }^{1}$, J. Jesús Vargas-Hernández ${ }^{{ }^{*}}$, Javier López-Upton ${ }^{1}$ \\ Armando Gómez-Guerrero ${ }^{1}$ y Humberto Vaquera-Huerta ${ }^{2}$
}

\author{
${ }^{1}$ Postgrado Forestal y ${ }^{2}$ Postgrado en Estadística, Colegio de Postgraduados, Campus Montecillo. Km 36.5 Carretera, México-Texcoco. 56320, Montecillo, \\ Texcoco, Estado de México. \\ * Autor para correspondencia (vargashj@colpos.mx)
}

\section{RESUMEN}

Con el propósito de identificar las posibilidades de adaptación de Pinus pinceana Gordon al estrés ambiental asociado con el cambio climático, se evaluó en invernadero el efecto del estrés hídrico y del estrés por altas temperaturas, sobre el crecimiento y distribución de biomasa en plantas de 12 procedencias o poblaciones de la especie. El diseño experimental incluyó dos ambientes de temperatura con diferencia promedio de $2{ }^{\circ} \mathrm{C}$, y dos niveles de disponibilidad de agua ( $S_{0}=$ potencial hídrico del suelo mayor a $-0.5 \mathrm{Mpa} ; \mathrm{S}_{1}=$ potencial hídrico del suelo inferior a $\mathbf{- 1 . 5} \mathrm{Mpa}$ ). Los dos factores ocasionaron una reducción $(P \leq \mathbf{0 . 0 5})$ en el crecimiento del brote, acumulación de biomasa y relación parte aérea-raíz, pero el efecto del estrés hídrico fue dos a tres veces mayor que el de la temperatura. El aumento en temperatura ocasionó mayor engrosamiento del tallo, efecto que fue más notorio al estar en combinación con estrés hídrico. Se encontró una amplia variación entre las procedencias en el potencial de crecimiento y en la asignación de biomasa en respuesta a los factores de estrés, lo que indica plasticidad fenotípica en sus mecanismos de adaptación. En condiciones de estrés, las procedencias de la región norte redujeron más la acumulación de biomasa y la relación parte aérea-raíz, pero produjeron más ramas que las del sur. Estos resultados permiten postular que esta especie tiene posibilidades de adaptarse al cambio climático, si las poblaciones con mejor respuesta al estrés se usan en programas de conservación y de restauración ecológica.

Palabras clave: Pinus pinceana, adaptación, distribución de biomasa, estrés hídrico, estrés térmico.

\section{SUMMARY}

To identify the potential for adaptability of Pinus pinceana Gordon to environmental stress associated with climate change, the effect of drought and high temperature stress on growth and biomass allocation in seedlings from 12 provenances of the species was evaluated under greenhouse conditions. The experimental design included two different temperature environments with an average difference of $2{ }^{\circ} \mathrm{C}$, and two levels of water availability $\left(\mathrm{S}_{0}=\right.$ soil water potential above $-0.5 \mathrm{Mpa} ; \mathrm{S}_{1}=$ soil water potential below -1.5 $M p a)$. Both factors caused a reduction $(P \leq 0.05)$ in shoot growth, biomass accumulation and shoot/root ratio, but the effect of water stress was two to three times higher than temperature stress. The increase in temperature caused a greater thickening of the stem,

Recibido: 15 de Julio del 2009.

Aceptado: 21 de Junio del 2010. particularly when combined with water stress. A wide variation among provenances in growth potential and biomass allocation was found in response to stress factors, indicating phenotypic plasticity in their adaptation mechanisms. Under stress conditions, populations from the northern region showed higher reductions in biomass accumulation and shoot/root ratio, but produced more branches than populations from the southern region. These results allow us to postulate that the species has the potential to adapt to climate change, if populations with best response to the predicted stress levels are used in conservation and ecological restoration programs.

Index words: Pinus pinceana, adaptation, biomass allocation, drought stress, heat stress.

\section{INTRODUCCIÓN}

Pinus pinceana Gordon, árbol piñonero endémico de México, crece en condiciones extremas de aridez a orillas del desierto en el noreste y centroeste del país, en bosques puros y a veces mezclados con $P$. cembroides Zucc. (Perry, 1991). Pinus pinceana es de importancia ecológica y económica para los habitantes de esas regiones como la principal fuente de leña y combustible, así como de piñones comestibles (piñón blanco) y forraje para animales domésticos (Ledig et al., 2001). Además, la especie es uno de los elementos estructurales clave de los ecosistemas de piñonares y de ella dependen muchas especies vegetales y animales que requieren de hábitat $o$ alimento.

La especie tiene una distribución natural limitada, por lo que está dentro de la lista de especies raras y amenazadas (Ramírez-Herrera et al., 2008). Se conocen menos de 15 poblaciones pequeñas, dispersas y discontinuas, que crecen en ambientes áridos de las laderas interiores de la Sierra Madre Oriental en los Estados de Coahuila, Zacatecas, San Luis Potosí, Querétaro e Hidalgo (Molina-Freaner et al., 2001; Ramírez-Herrera et $a l ., 2008$ ). La mayoría de las poblaciones se encuentran amenazadas por el pastoreo, los incendios y la extracción 
de leña $\mathrm{y}$ frutos. La tendencia en el aumento de temperatura y sequía asociadas con el cambio climático amenaza a las poblaciones localizadas en los ambientes de mayor aridez. Sin embargo, no existe suficiente información para establecer un adecuado programa de manejo y conservación (Molina-Freaner et al., 2001).

El aislamiento geográfico y el gradiente ambiental sugieren la posibilidad de que ocurran diferencias entre las poblaciones en características de importancia adaptativa a sequía y temperaturas elevadas. En la región norte, en los Estados de Coahuila y Zacatecas, $P$. pinceana presenta mayor contenido de cera en las hojas que en los Estados de Querétaro e Hidalgo, ubicados en el sur (Ramírez-Herrera, Com. personal ${ }^{1}$ ). Este autor encontró una relación positiva entre el contenido de cera y la materia seca del follaje, que puede indicar que las poblaciones han desarrollado estrategias para adaptarse a las condiciones restrictivas del medio en que evolucionaron. Sin embargo, se carece de información sobre la plasticidad fenotípica y los mecanismos de adaptación de la especie a condiciones inducidas de estrés hídrico y altas temperaturas, así como del grado de diferenciación poblacional en esta respuesta.

No existen datos sobre el crecimiento y distribución de biomasa de $P$. pinceana en condiciones de sequía o temperatura elevada, mecanismos adaptativos importantes ante el cambio climático. Córdoba et al. (2008) encontraron una amplia plasticidad fenotípica en el crecimiento de la raíz de $P$. pinceana en respuesta al tipo de suelo, y aunque hubo diferencias entre poblaciones en el crecimiento de las plantas, el patrón de asignación de biomasa fue similar entre ellas. La asignación de biomasa a la parte aérea y a la raíz está fuertemente asociada con la productividad y la capacidad de adaptación a diferentes condiciones de estrés. Zhang et al. (1996), por ejemplo, encontraron diferencias entre poblaciones de Pseudotsuga menziesii (Mirb.) Franco, Pinus ponderosa Dougl. ex Laws. y Larix occidentalis Nutt. en la asignación de materia seca al someterlas a condiciones de sequía.

En este trabajo se evaluó el efecto del estrés por sequía y por temperatura sobre el crecimiento y distribución de biomasa en plantas de $P$. pinceana, con el propósito de determinar las posibles diferencias adaptativas entre procedencias o poblaciones, en respuesta a los dos tipos de estrés. Se postula que la información permitirá entender los mecanismos de adaptación de la especie a factores específicos y obtener datos para la restauración y

${ }^{1}$ Ramírez-Herrera C (2007) Quantitative trait variation and allozyme diversity of Pinus pinceana. Ph. D. Thesis in Forestry. University of New Brunswick, Canada. 213 p. manejo de sus poblaciones ante las expectativas de cambio climático. Conforme aumente la severidad de las sequías y altas temperaturas en las regiones semi-áridas de México, $P$. pinceana será una de las pocas especies de coníferas con potencial para reforestación en esas áreas.

\section{MATERIALES Y MÉTODOS}

\section{Establecimiento del ensayo}

En el ensayo se evaluaron plantas de 12 procedencias de $P$. pinceana producidas a partir de semilla colectada en todas las áreas de distribución de la especie (Figura 1). Siete poblaciones provienen de la región norte y cinco de la sur (Cuadro 1). El experimento se estableció en un invernadero en Montecillo, Texcoco, Edo. de México (19 $27^{\prime} 38^{\prime \prime}$ LN, 98 24' 54' ' LO, $\left.2250 \mathrm{~m}\right)$, en marzo de 2007. Cada población estuvo representada por 10 familias de polinización libre. Se utilizaron plantas de 25 meses de edad crecidas en contenedores de $350 \mathrm{~cm}^{3}$ en un sustrato formado por corteza de pino, agrolita y suelo agrícola arenoso en proporción 25:25:50. Se seleccionaron plantas de tamaño homogéneo en cada población y se trasplantaron a envases de $1500 \mathrm{~cm}^{3}$ rellenados con el mismo sustrato. Se hicieron pruebas físicas para determinar las constantes de humedad del sustrato [capacidad de campo (-0.03 Mpa) y punto de marchitamiento permanente $(-1.5 \mathrm{Mpa})$ ], y en un ensayo previo se definió el nivel de humedad requerido para inducir estrés por sequía.

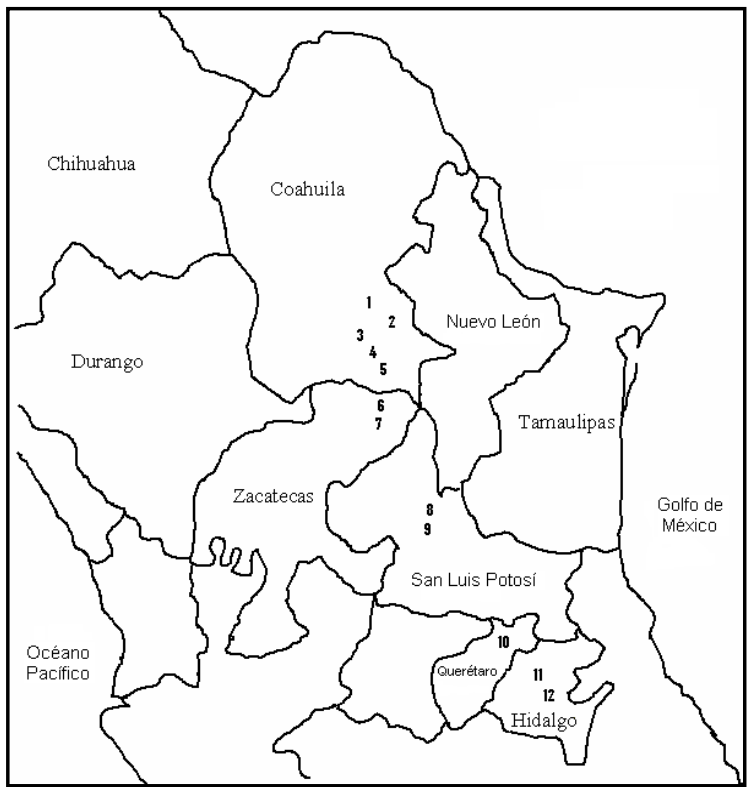

Figura 1. Mapa de distribución de las procedencias de Pinus pinceana usadas en el ensayo: 1. Norias; 2. El Recreo; 3. El Cinco; 4. Palmas Altas; 5. Santa Elena; 6. El Orégano; 7. Carbonerillas; 8. Matehualilla; 9. Ejido Núñez; 10. Maguey Verde; 11. El Arenalito; 12. San Cristóbal. 


\begin{tabular}{|c|c|c|c|c|}
\hline \multirow{2}{*}{$\begin{array}{l}\text { Núm. de } \\
\text { control }\end{array}$} & \multirow{2}{*}{$\begin{array}{c}\text { Población } \\
\text { (Procedencia) }\end{array}$} & \multicolumn{2}{|c|}{ Coordenadas geográficas } & \multirow{2}{*}{$\begin{array}{l}\text { Altitud } \\
\text { (m) }\end{array}$} \\
\hline & & Latitud (N) & Longitud $(\mathrm{O})$ & \\
\hline \multicolumn{5}{|c|}{ Región Norte } \\
\hline 1 & Norias, Coah. & $26^{\circ} 27^{\prime} 08^{\prime \prime}$ & $101^{\circ} 35^{\prime} 40^{\prime \prime}$ & 1800 \\
\hline 2 & El Recreo, Coah. & $25^{\circ} 17^{\prime} 40^{\prime \prime}$ & $101^{\circ} 00^{\prime} 02^{\prime \prime}$ & 2238 \\
\hline 3 & El Cinco, Coah. & $25^{\circ} 10^{\prime} 41^{\prime \prime}$ & $101^{\circ} 41^{\prime} 16^{\prime \prime}$ & 2250 \\
\hline 4 & Palmas Altas, Coah. & $25^{\circ} 07^{\prime} 57^{\prime \prime}$ & $101^{\circ} 27^{\prime} 04^{\prime \prime}$ & 2090 \\
\hline 5 & Santa Elena, Coah. & $25^{\circ} 01^{\prime} 38^{\prime \prime}$ & $101^{\circ} 24^{\prime} 29^{\prime \prime}$ & 2077 \\
\hline 6 & El Orégano, Zac. & $24^{\circ} 30^{\prime} 19^{\prime \prime}$ & $101^{\circ} 27^{\prime} 48^{\prime \prime}$ & 2305 \\
\hline 7 & Carbonerillas, Zac. & $24^{\circ} 28^{\prime} 16^{\prime \prime}$ & $100^{\circ} 26^{\prime} 53^{\prime \prime}$ & 2300 \\
\hline \multicolumn{5}{|c|}{ Región Sur } \\
\hline 8 & Matehualilla, S.L.P. & $22^{\circ} 42^{\prime} 32^{\prime \prime}$ & $100^{\circ} 28^{\prime} 05^{\prime \prime}$ & 2020 \\
\hline 9 & Ejido Núñez, S.L.P. & $22^{\circ} 40^{\prime} 06^{\prime \prime}$ & $100^{\circ} 28^{\prime} 24^{\prime \prime}$ & 1930 \\
\hline 10 & Maguey Verde, Qro. & $21^{\circ} 05^{\prime} 25^{\prime \prime}$ & $099^{\circ} 42^{\prime} 00^{\prime \prime}$ & 2176 \\
\hline 11 & El Arenalito, Hgo. & $20^{\circ} 39^{\prime} 27^{\prime \prime}$ & $099^{\circ} 02^{\prime} 58^{\prime \prime}$ & 1880 \\
\hline 12 & San Cristóbal, Hgo. & $20^{\circ} 37^{\prime} 53^{\prime \prime}$ & $098^{\circ} 58^{\prime} 31^{\prime \prime}$ & 1915 \\
\hline
\end{tabular}

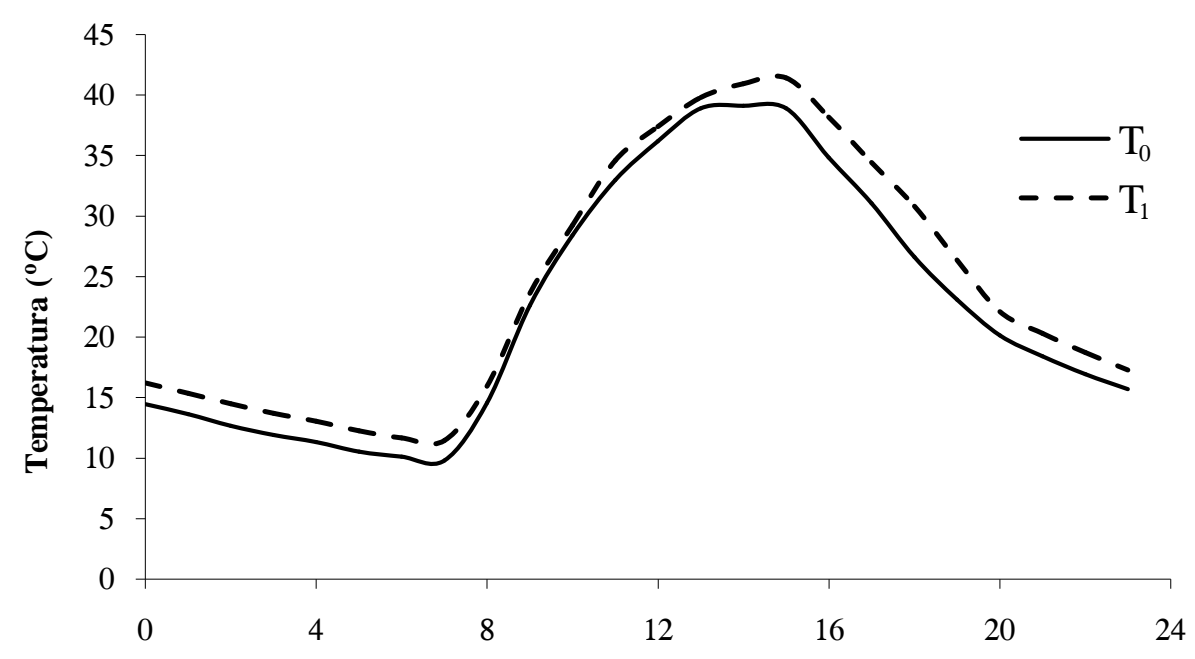

Hora del día

Figura 2. Fluctuación típica de temperatura a lo largo del día en las dos condiciones del experimento (To $=$ testigo; $\mathbf{T}_{1}=$ temperatura elevada).

Para generar dos ambientes de temperatura, el invernadero se separó en dos secciones; una de ellas con dos calefactores portátiles y la otra sin calefactores (testigo). En cada sección se registró la temperatura del aire mediante sensores electrónicos con capacidad de almacenamiento de datos (Hanna Instruments, modelo HI141JH® Padua, Italia. En el ambiente testigo ( $\left.\mathrm{T}_{0}\right)$ se tuvo una temperatura promedio de $29.5^{\circ} \mathrm{C}$ en el día y $14.9{ }^{\circ} \mathrm{C}$ en la noche, mientras que en el otro ambiente $\left(\mathrm{T}_{1}\right)$ se tuvo una temperatura promedio de $31.3{ }^{\circ} \mathrm{C}$ en el día y de 16.8 ${ }^{\circ} \mathrm{C}$ en la noche, con una diferencia promedio de $2{ }^{\circ} \mathrm{C}$ entre ellos (Figura 2).

\section{Diseño experimental}

Con el propósito de separar el efecto del estrés hídrico del causado por altas temperaturas, se utilizó un diseño factorial de parcelas divididas en dos ambientes (temperatura), con cuatro repeticiones por tratamiento. En cada temperatura los tratamientos de humedad se asignaron a las parcelas grandes y las poblaciones $\mathrm{o}$ procedencias a las parcelas pequeñas, con las repeticiones anidadas dentro de cada temperatura. Cada unidad experimental estuvo representada por cinco plantas. Al inicio del ensayo, todas las plantas se regaron a capacidad de campo; en el testigo ( $\mathrm{S}_{0}=$ humedad favorable) cada 6 
d se repuso el agua perdida por cada planta, mediante el método gravimétrico; en estrés hídrico $\left(\mathrm{S}_{1}=\right.$ sequía $)$ se permitió la desecación del suelo hasta que el contenido de humedad llegó a $12 \%$ con relación a la masa, momento en que se agregó agua para llevarlo a $20 \%$ (Figura 3), de acuerdo con las condiciones establecidas en el ensayo previo; estas condiciones de humedad se mantuvieron durante $160 \mathrm{~d}$.

\section{Variables de respuesta}

Al inicio del ensayo se midió la altura del tallo, el diámetro en la base del tallo y el número de ramas en cada planta. Al finalizar el experimento se evaluaron nuevamente las variables anteriores, además de la biomasa de raíz, tallo y hojas. Para obtener la biomasa, las muestras se secaron en un horno eléctrico a $70{ }^{\circ} \mathrm{C}$ por $3 \mathrm{~d}$, hasta que alcanzaron peso constante. Con la diferencia entre el valor inicial y el valor final respectivo, se obtuvo el incremento en altura, diámetro y número de ramas de cada planta durante el periodo de estudio. Con los datos de biomasa se obtuvo la biomasa total de la planta y la relación parte aérea/raíz.

\section{Análisis estadístico}

Previo al análisis de varianza, se revisó que las variables cumplieran el supuesto de normalidad; en el caso del número de ramas se utilizó la transformación raíz cuadrada. Un análisis de varianza con un modelo que incluyó sólo el efecto de los factores temperatura y sequía (i.e., sin incluir el efecto de procedencias), mostró que en la mayoría de las características no hubo interacción significativa entre estos factores. Por tanto, en adición al anterior se realizó un análisis de varianza de la respuesta de las plantas con un modelo estadístico que incluyó los factores temperatura, sequía, procedencias y sus interacciones. En el análisis, hecho con el paquete estadístico SAS (SAS Institute, 1988), se utilizaron los valores promedio por parcela y se consideró a todos los factores como de efectos fijos. Cuando se detectaron diferencias significativas entre tratamientos o procedencias, la comparación de medias se hizo con la prueba de comparaciones múltiples de Duncan $(\alpha=0.05)$. Para estimar las diferencias entre procedencias en la respuesta a las condiciones de estrés, se calculó el cociente del valor promedio de la característica en el tratamiento de estrés $\left(\mathrm{T}_{1}, \mathrm{~S}_{1}\right)$ entre el valor del testigo respectivo $\left(\mathrm{T}_{0}\right.$, $\mathrm{S}_{0}$ ). Este cociente representa el cambio relativo en las características promedio de las plantas al pasar de un ambiente a otro. De la misma manera, para evaluar la relación entre la respuesta de las poblaciones $\mathrm{o}$ procedencias a las condiciones de estrés con el potencial de crecimiento, se estimó la correlación de las diferentes características en condiciones de estrés térmico o hídrico con el peso seco de las plantas en condiciones favorables $\left(\mathrm{T}_{0} \mathrm{~S}_{0}\right)$.

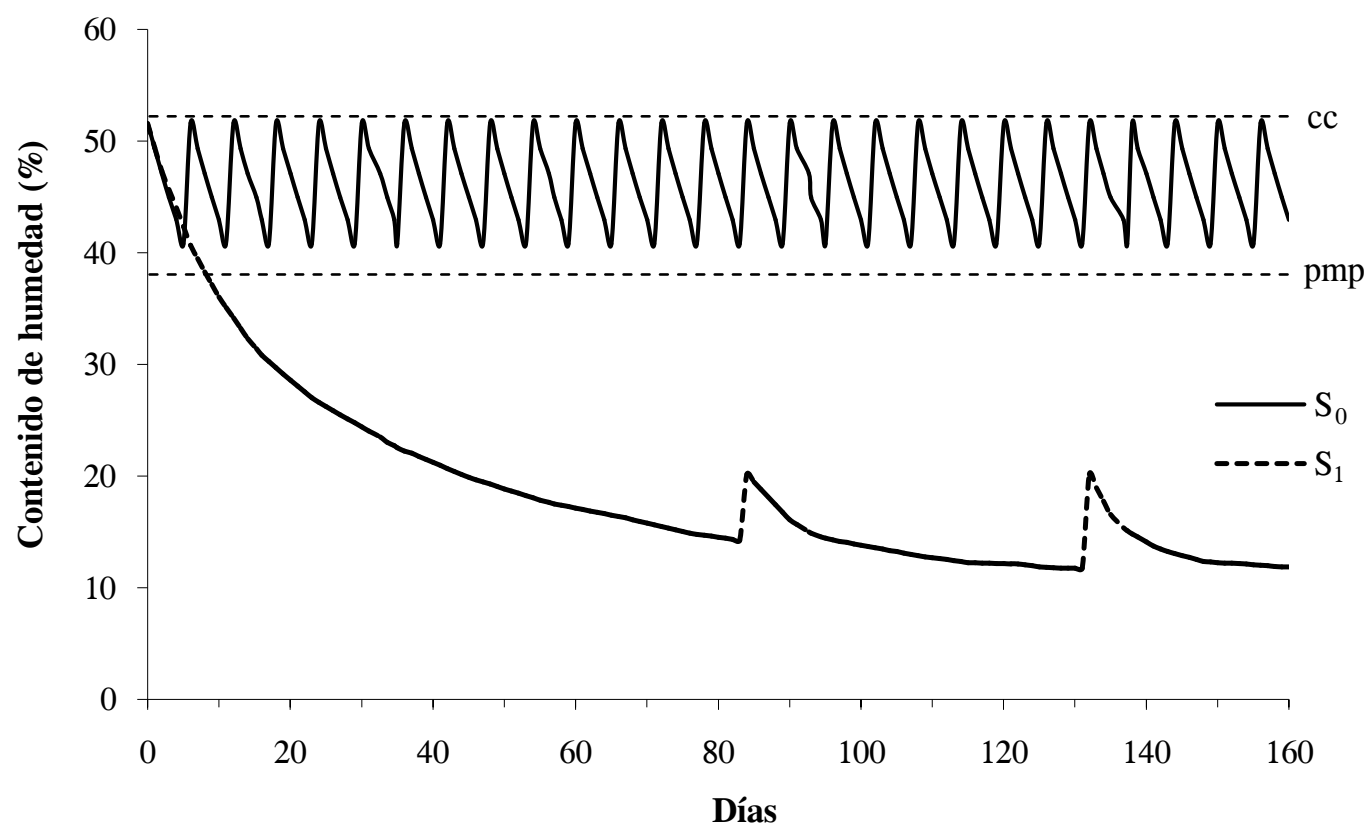

Figura 3. Contenido de humedad del suelo en los tratamientos de riego $\left(\mathrm{S}_{0}\right)$ y sequía $\left(\mathrm{S}_{1}\right)$ durante el ensayo [cc $=$ capacidad de campo, -0.03 MPa; pmp = punto de marchitamiento permanente, $-1.5 \mathrm{MPa}$. 


\section{RESULTADOS Y DISCUSIÓN}

\section{Efecto del estrés por temperatura}

Se encontraron diferencias $(\mathrm{P} \leq 0.05)$ entre los dos ambientes de temperatura en el incremento en altura y diámetro del tallo, así como en el peso seco de hojas, biomasa total y relación parte aérea-raíz (Cuadro 2). Las plantas disminuyeron su crecimiento en condiciones de temperatura elevada, con excepción del diámetro del tallo, en donde se observó un crecimiento $27 \%$ mayor (Cuadro 3). La acumulación de biomasa total se redujo en $7 \%$ por efecto de la temperatura, pero la reducción fue mayor en la parte aérea que en la raíz, lo que ocasionó una reducción en la relación parte aérea-raíz (Cuadro 3).

Es decir, un aumento en la temperatura promedio de 2 ${ }^{\circ} \mathrm{C}$ fue suficiente para causar una reducción en la tasa de crecimiento de las plantas de $P$, pinceana en un periodo relativamente reducido $(160 \mathrm{~d})$, lo que indica la importancia del estrés inducido por este factor. Diversos autores han reconocido que la temperatura supraóptima afecta negativamente diferentes procesos fisiológicos en las plantas, como fotosíntesis, respiración y uso del agua (Kozlowski, 1979; Teskey y Will, 1999), así como el metabolismo de los ácidos nucléicos y síntesis de proteínas (McDaniel, 1982), lo que se refleja en un descenso en la acumulación de biomasa. La reducción en la relación parte aérea-raíz indica un mecanismo de adaptación al estrés para mantener una relación más favorable entre la absorción de agua y la transpiración, que permita a las plantas un mejor balance de la carga energética en las hojas.

Según Teskey y Will (1999), las temperaturas elevadas originaron un descenso en el crecimiento en altura y en el acopio de biomasa de $P$. taeda L., que los autores atribuyeron a la disminución de la capacidad fotosintética de las hojas y al aumento de la respiración. Por el contrario, Cantin et al. (1997) encontraron que la biomasa de $P$. banksiana Lamb. aumentó con temperaturas y niveles de $\mathrm{CO}_{2}$ elevados, y Zha et al. (2001) observaron efectos similares en $P$. silvestris L. Sin embargo, es posible que las temperaturas que ellos utilizaron no fueran suficientes para provocar estrés en las plantas, ya que no excedieron de $30{ }^{\circ} \mathrm{C}$. Además, el aumento del $\mathrm{CO}_{2}$ del aire ocasiona ganancias en la biomasa de plantas $\mathrm{C}_{3}$ cuando la disponibilidad de agua y nutrientes no llega a un nivel crítico (Schlesinger y Lichter, 2001).

Contrario a lo esperado, el aumento en temperatura ocasionó mayor engrosamiento del tallo de las plantas de $P$. pinceana. Kolb y Robberecht (1996) encontraron un comportamiento similar en plantas de $P$. ponderosa, que ellos interpretaron como un mecanismo de adaptación para soportar las altas temperaturas, al aumentar la superficie de conducción de agua en el xilema y así ocasionar mayor efecto de enfriamiento en la planta.

Cuadro 2. Resultados del análisis de varianza (significancia estadística) para las características de crecimiento de las plantas de varias procedencias de Pinus pinceana, en respuesta a los factores de estrés por temperatura y sequía.

\begin{tabular}{|c|c|c|c|c|c|c|c|c|c|}
\hline \multirow{2}{*}{ Fuente de variación } & \multirow{2}{*}{$\mathrm{gl}$} & \multicolumn{3}{|c|}{ Incremento } & \multicolumn{4}{|c|}{ Biomasa } & \multirow{2}{*}{$\begin{array}{c}\text { Relación parte } \\
\text { aérea/raíz }\end{array}$} \\
\hline & & Altura & Diámetro & Número de ramas & Tallo & Hojas & Raíz & Total & \\
\hline Temperatura (T) & 1 & $* *$ & $* *$ & $\mathrm{~ns}$ & ns & $*$ & ns & $*$ & $* *$ \\
\hline Sequía $(S)$ & 1 & $* *$ & $* *$ & $* *$ & $* *$ & $* *$ & $* *$ & $* *$ & $* *$ \\
\hline $\mathrm{T} \times \mathrm{S}$ & 1 & $\mathrm{~ns}$ & $* *$ & $\mathrm{~ns}$ & $\mathrm{~ns}$ & $\mathrm{~ns}$ & $\mathrm{~ns}$ & $\mathrm{~ns}$ & $\mathrm{~ns}$ \\
\hline Procedencia $(\mathrm{P})$ & 11 & $* *$ & $* *$ & $* *$ & $* *$ & $* *$ & $* *$ & $* *$ & $* *$ \\
\hline $\mathrm{T} \times \mathrm{P}$ & 11 & $* *$ & $* *$ & ns & ns & $\mathrm{ns}$ & $*$ & $\mathrm{~ns}$ & $*$ \\
\hline $\mathrm{S} \times \mathrm{P}$ & 11 & $* *$ & $* *$ & $*$ & $* *$ & $*$ & $* *$ & $* *$ & $*$ \\
\hline$T \times S \times P$ & 11 & $* *$ & $* *$ & $\mathrm{~ns}$ & $\mathrm{~ns}$ & $* *$ & $\mathrm{~ns}$ & $*$ & $\mathrm{~ns}$ \\
\hline
\end{tabular}

*,** Diferencias significativas con $\mathrm{P} \leq 0.05$ y $\mathrm{P} \leq 0.01$, respectivamente; $\mathrm{ns}=$ no significativo con $\mathrm{P}=0.05 ; \mathrm{gl}=$ grados de libertad.

Cuadro 3. Valores promedio de las características de crecimiento de las plantas de Pinus pinceana en las condiciones de temperatura ambiente $\left(\mathbf{T}_{0}\right)$ y temperatura elevada $\left(\mathbf{T}_{1}\right)$.

\begin{tabular}{|c|c|c|c|c|c|c|c|c|}
\hline \multirow[b]{2}{*}{ Temp. } & \multicolumn{3}{|c|}{ Incremento } & \multicolumn{4}{|c|}{ Biomasa (g) } & \multirow[b]{2}{*}{$\begin{array}{l}\text { Relación parte } \\
\text { aérea/raíz }\end{array}$} \\
\hline & $\begin{array}{c}\text { Altura } \\
(\mathrm{cm})\end{array}$ & $\begin{array}{c}\text { Diámetro } \\
(\mathrm{mm})\end{array}$ & $\begin{array}{l}\text { Número de } \\
\text { ramas }\end{array}$ & Tallo & Hojas & Raíz & Total & \\
\hline $\mathrm{T}_{0}$ & $2.84 \mathrm{a}$ & $0.30 \mathrm{~b}$ & $1.29 \mathrm{a}$ & $2.36 \mathrm{a}$ & $3.70 \mathrm{a}$ & $3.27 \mathrm{a}$ & $9.34 \mathrm{a}$ & $1.91 \mathrm{a}$ \\
\hline $\mathrm{T}_{1}$ & $2.10 \mathrm{~b}$ & $0.38 \mathrm{a}$ & $0.87 \mathrm{a}$ & $2.24 \mathrm{a}$ & $3.24 \mathrm{~b}$ & $3.22 \mathrm{a}$ & $8.70 \mathrm{~b}$ & $1.76 \mathrm{~b}$ \\
\hline
\end{tabular}

Medias en una misma columna con letras iguales no son estadísticamente diferentes (Duncan, 0.05). 


\section{Efecto del estrés hídrico}

Todas las variables evaluadas mostraron diferencias ( $\mathrm{P}$ $\leq 0.05$ ) entre las dos condiciones de humedad (Cuadro 2). La sequía afectó más al crecimiento de las plantas que el estrés por temperatura; así, el incremento en altura se redujo en más de $80 \%$, el incremento en diámetro en 49 $\%$ y en el número de ramas en casi $60 \%$ (Cuadro 4). La acumulación de biomasa se redujo entre 15 y $30 \%$; la parte aérea fue más afectada que la raíz, lo que causó una reducción de $15 \%$ en la relación parte aérea-raíz (Cuadro 4). Estudios en especies leñosas han mostrado que uno de los principales efectos de la sequía es la reducción del crecimiento, aunque la severidad del efecto ha dependido de la duración e intensidad del estrés hídrico y de los mecanismos adaptativos de la especie (Samuelson y Seiler, 1993; Ngugi et al., 2003; Susiluoto y Berninger, 2007).

La reducción de la relación parte aérea-raíz es un mecanismo que utilizan las plantas en condiciones de estrés hídrico para mantener un equilibrio más favorable entre la capacidad de absorción y uso del agua (Cregg, 1994). Al igual que en este estudio, Mcmillin y Wagner (1995) y Susiluoto y Berninger (2007) encontraron una reducción de la relación parte aérea-raíz en plantas de $P$. ponderosa y Eucalyptus microtheca F. Muell., respectivamente. En Gliricidia sepium (Jacq.) Kunth ex Walp (García-Figueroa y Vargas-Hernández, 2000), Pinus greggii Engelm. (Hernández et al., 2001) y P. leiophylla Schl. et Cham. (Martínez et al., 2002), también se ha detectado reducción de la relación parte aérea-raíz al ser expuestas a estrés hídrico, aunque la magnitud difiere entre especies.

\section{Interacción entre los dos tipos de estrés}

En la mayoría de las características no se encontró un efecto significativo de la interacción entre los dos factores de estrés sobre el crecimiento de las plantas (Cuadro 2), lo que indica que el efecto de cada uno fue independiente. Sin embargo, en el caso del diámetro del tallo el aumento relativo ocasionado por la temperatura elevada fue mayor en las plantas sometidas a estrés hídrico que bajo condiciones favorables de humedad (Figura 4); al respecto se ha postulado que la mayor necesidad de enfriamiento ocasionada por el estrés hídrico estimula en mayor grado el engrosamiento del tallo para aumentar así la eficiencia de conducción de agua en el xilema, como se ha reportado para Pinus ponderosa (Kolb y Robberecht, 1996).

Cuadro 4. Valores promedio de las características de crecimiento de las plantas de Pinus pinceana en condiciones de humedad favorable $\left(\mathbf{S}_{0}\right)$ y estrés por sequía $\left(\mathbf{S}_{1}\right)$.

\begin{tabular}{cccccccc}
\hline Trat. & \multicolumn{3}{c}{ Incremento } & \multicolumn{3}{c}{ Biomasa $(\mathrm{g})$} \\
\cline { 2 - 7 } & $\begin{array}{c}\text { Altura } \\
(\mathrm{cm})\end{array}$ & $\begin{array}{c}\text { Diámetro } \\
(\mathrm{mm})\end{array}$ & $\begin{array}{c}\text { Número de } \\
\text { ramas }\end{array}$ & Tallo & Hojas & Raíz & Total \\
aérea/raíz & $1.54 \mathrm{a}$ & $2.70 \mathrm{a}$ & $3.94 \mathrm{a}$ & $3.49 \mathrm{a}$ & $10.13 \mathrm{a}$ \\
$\mathrm{S}_{0}$ & $4.16 \mathrm{a}$ & $0.45 \mathrm{a}$ & $1.98 \mathrm{a}$ & $3.00 \mathrm{~b}$ & $7.91 \mathrm{~b}$ \\
$\mathrm{~S}_{1}$ & $0.79 \mathrm{~b}$ & $0.23 \mathrm{~b}$ & $0.63 \mathrm{~b}$ & $1.90 \mathrm{~b}$ & $3.01 \mathrm{~b}$ & $1.69 \mathrm{~b}$ \\
\hline
\end{tabular}

Medias en una misma columna con letras iguales no son estadísticamente diferentes (Duncan, 0.05).

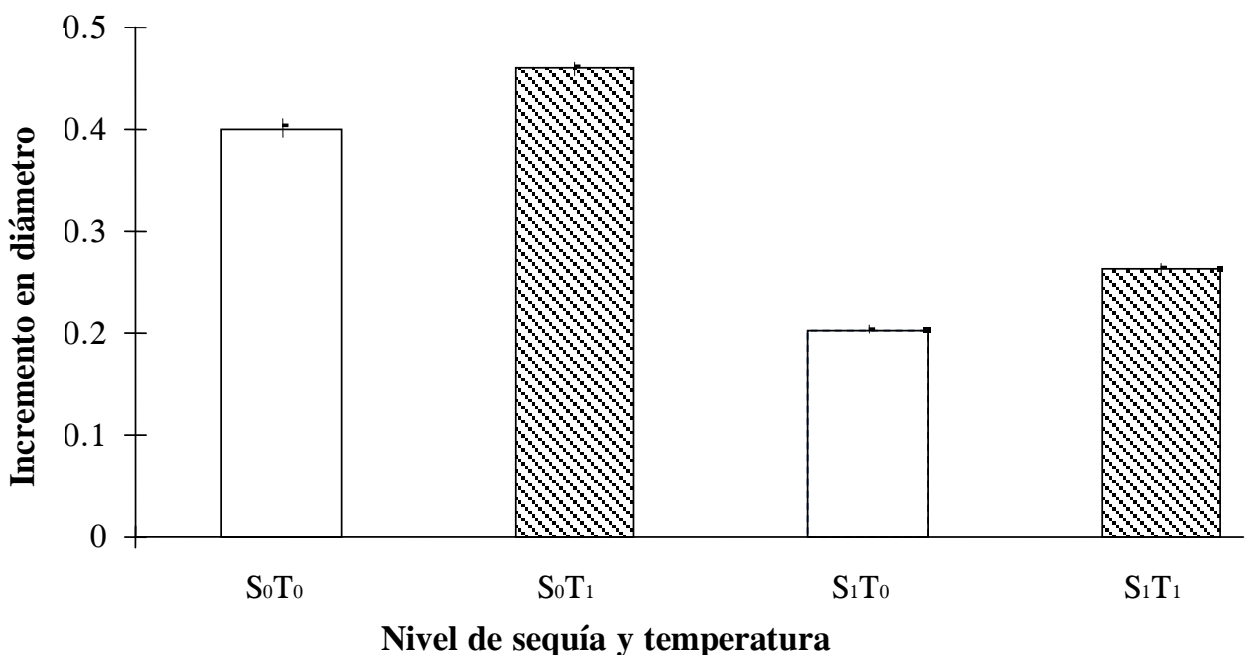

Figura 4. Interacción del estrés por sequía $\left(S_{1}\right)$ y por temperatura $\left(T_{1}\right)$ sobre el incremento promedio en diámetro de las plantas de Pinus pinceana con respecto al crecimiento en condiciones favorables de humedad ( $\left.\mathrm{S}_{\mathrm{o}}\right)$ y temperatura $\left(\mathbf{T}_{\mathbf{o}}\right)$. 


\section{Diferencias entre poblaciones en el crecimiento de las plantas}

Se encontró una amplia variación en el crecimiento de las plantas entre las procedencias estudiadas, con diferencias $(\mathrm{P}<0.01)$ entre ellas en todas las características de crecimiento (Cuadro 2). Las diferencias extremas entre procedencias fluctuaron desde $19 \%$ en la biomasa total, hasta casi $60 \%$ en el número de ramas, lo que indica la gran variabilidad geográfica existente en esta especie en cuanto al potencial de crecimiento y otras características de importancia adaptativa (Cuadro 5). Así, la población de Carbonerillas presentó la menor relación parte aérea-raíz y el mayor incremento en altura, mientras que la de Maguey Verde presentó uno de los mayores valores en la relación parte aérea-raíz y uno de los menores incrementos en altura. En promedio, las poblaciones del sur acumularon mayor biomasa de tallo y total, y presentaron una mayor relación parte aérea-raíz que las poblaciones del norte, pero tuvieron menor incremento en altura y en ramas que éstas últimas (Cuadro 5). Estos resultados coinciden con los datos obtenidos por Ramírez-Herrera (Com. personal ${ }^{2}$ ) en un estudio realizado con las mismas poblaciones de la especie, en donde se encontró que las poblaciones de la región norte acumularon menos biomasa que las del sur.

Estudios previos realizados en otras especies señalan que el patrón de asignación de biomasa es una caracterís- tica de importancia adaptativa a condiciones de estrés. Por ejemplo, Zhang et al. (1996) encontraron diferencias significativas entre poblaciones de Pseudotsuga menziesii y Pinus ponderosa en la relación parte aérea-raíz; en ambos casos las poblaciones de sitios secos presentaron una menor relación parte aérea-raíz. García-Figueroa y Vargas-Hernández (2000) también encontraron diferencias significativas entre poblaciones en el patrón de asignación de biomasa de ocho procedencias de semilla de Gliricidia sepium. Los resultados del presente estudio muestran que las poblaciones de $P$. pinceana de la región norte presentan el síndrome característico de adaptación a condiciones más severas de sequía, al mantener una menor relación parte aérea-raíz y una mayor proliferación de ramas, con menor biomasa.

\section{Variación en la respuesta de las poblaciones al estrés por temperatura}

Las poblaciones respondieron de manera diferente a los dos regímenes de temperatura en cuanto al incremento en altura y en diámetro, así como en la acumulación de biomasa en raíz y en la relación parte aérea-raíz (Cuadro 2). A pesar de que la respuesta promedio en las dos regiones geográficas fue similar en la mayoría de las variables medidas, las poblaciones del norte mostraron mayor engrosamiento del tallo en respuesta al aumento de la temperatura (Cuadro 6).

Cuadro 5. Valores promedio por procedencia (población) de las características de crecimiento de las plantas de Pinus pinceana al crecer en dos condiciones de temperatura y humedad.

\begin{tabular}{|c|c|c|c|c|c|c|c|c|c|c|c|c|c|c|c|c|}
\hline \multirow{3}{*}{$\begin{array}{l}\text { Población } \\
\text { (procedencia) }\end{array}$} & \multicolumn{6}{|c|}{ Incremento en } & \multicolumn{8}{|c|}{ Biomasa (g) } & \multirow{2}{*}{\multicolumn{2}{|c|}{$\begin{array}{l}\text { Relación } \\
\text { parte } \\
\text { aérea/raíz }\end{array}$}} \\
\hline & \multicolumn{2}{|c|}{$\begin{array}{l}\text { Altura } \\
(\mathrm{cm})\end{array}$} & \multicolumn{2}{|c|}{$\begin{array}{c}\text { Diámetro } \\
(\mathrm{mm})\end{array}$} & \multicolumn{2}{|c|}{$\begin{array}{l}\text { Número de } \\
\text { ramas }\end{array}$} & \multicolumn{2}{|c|}{ Tallo } & \multicolumn{2}{|c|}{ Hojas } & \multicolumn{2}{|c|}{ Raíz } & \multicolumn{2}{|c|}{ Total } & & \\
\hline & 2.38 & cde & 0.35 & $\mathrm{e}$ & 1.70 & $\mathrm{a}$ & 2.14 & cde & 3.21 & cde & 3.22 & bcd & 8.57 & $\mathrm{~cd}$ & 1.69 & def \\
\hline El Recreo & 2.90 & $\mathrm{ab}$ & 0.32 & $\mathrm{f}$ & 1.23 & bc & 2.13 & cde & 3.63 & $\mathrm{ab}$ & 3.34 & bc & 9.11 & $\mathrm{~cd}$ & 1.76 & cdef \\
\hline El Cinco & 2.60 & bcd & 0.29 & g & 1.08 & bcd & 2.16 & cde & 3.70 & $\mathrm{ab}$ & 3.35 & $\mathrm{bc}$ & 9.21 & $\mathrm{bc}$ & 1.81 & cde \\
\hline Palmas Altas & 2.76 & bc & 0.24 & $\mathrm{~h}$ & 0.70 & $\mathrm{~d}$ & 1.97 & $\mathrm{e}$ & 3.39 & bcd & 3.33 & $\mathrm{bc}$ & 8.69 & $\mathrm{~cd}$ & 1.65 & ef \\
\hline Santa Elena & 2.61 & bcd & 0.46 & $\mathrm{a}$ & 1.05 & bcd & 2.07 & de & 3.46 & $a b c$ & 3.10 & bcde & 8.64 & $\mathrm{~cd}$ & 1.79 & cde \\
\hline El Orégano & 2.94 & $a b$ & 0.38 & $\mathrm{c}$ & 1.33 & $\mathrm{ab}$ & 2.21 & bcd & 3.65 & $a b$ & 3.25 & bcd & 9.11 & $\mathrm{~cd}$ & 1.85 & bcd \\
\hline Carbonerillas & 3.25 & $\mathrm{a}$ & 0.33 & $\mathrm{f}$ & 1.18 & $\mathrm{bc}$ & 2.00 & de & 3.11 & de & 3.25 & bcd & 8.36 & d & 1.62 & f \\
\hline$\overline{\mathbf{X}}$ Región Norte & 2.78 & & 0.34 & & 1.18 & & 2.10 & & 3.45 & & 3.26 & & 8.81 & & 1.74 & \\
\hline Matehualilla & 2.22 & def & 0.34 & $\mathrm{e}$ & 1.06 & bcd & 2.38 & $\mathrm{~b}$ & 3.37 & bcd & 2.79 & $\mathrm{e}$ & 8.54 & & 2.14 & $\mathrm{a}$ \\
\hline Ejido Núñez & 2.16 & ef & 0.42 & $\mathrm{~b}$ & 0.85 & $\mathrm{~cd}$ & 2.32 & bc & 3.03 & e & 2.96 & de & 8.31 & d & 1.91 & bc \\
\hline Maguey Verde & 2.06 & ef & 0.30 & g & 0.78 & $\mathrm{~cd}$ & 2.64 & $\mathrm{a}$ & 3.66 & $\mathrm{ab}$ & 3.04 & cde & 9.34 & $\mathrm{bc}$ & 2.12 & $\mathrm{a}$ \\
\hline El Arenalito & 2.11 & ef & 0.30 & g & 1.10 & bcd & 2.76 & $\mathrm{a}$ & 3.64 & $a b$ & 3.84 & $\mathrm{a}$ & 10.24 & $\mathrm{a}$ & 1.74 & def \\
\hline San Cristóbal & 1.89 & $\mathrm{f}$ & 0.37 & d & 0.88 & bcd & 2.72 & $\mathrm{a}$ & 3.79 & $\mathrm{a}$ & 3.43 & $\mathrm{~b}$ & 9.94 & $a b$ & 1.96 & $\mathrm{~b}$ \\
\hline$\overline{\mathbf{X}}$ Región Sur & 2.09 & & 0.35 & & 0.93 & & 2.56 & & 3.50 & & 3.21 & & 9.27 & & 1.97 & \\
\hline
\end{tabular}

Medias en una misma columna con letras iguales no son estadísticamente diferentes (Duncan, 0.05).

${ }^{2}$ Ramírez-Herrera C (2007). Op. cit. 


\begin{tabular}{|c|c|c|c|c|c|c|c|c|c|c|c|c|}
\hline \multirow[t]{2}{*}{$\begin{array}{l}\text { Procedencia } \\
\text { (población) }\end{array}$} & \multicolumn{3}{|c|}{$\begin{array}{l}\text { Incremento en altura } \\
(\mathrm{cm})\end{array}$} & \multicolumn{3}{|c|}{$\begin{array}{l}\text { Incremento en diámetro } \\
(\mathrm{mm})\end{array}$} & \multicolumn{3}{|c|}{$\begin{array}{c}\text { Biomasa de raíz } \\
(\mathrm{g})\end{array}$} & \multicolumn{3}{|c|}{$\begin{array}{l}\text { Relación parte } \\
\text { aérea/raíz }\end{array}$} \\
\hline & $\mathrm{T}_{0}$ & $\mathrm{~T}_{1}$ & $\overline{\mathrm{T}_{1} / \mathrm{T}_{0}}$ & $\mathrm{~T}_{0}$ & $\mathrm{~T}_{1}$ & $\mathrm{~T}_{1} / \mathrm{T}_{0}$ & $\mathrm{~T}_{0}$ & $\mathrm{~T}_{1}$ & $\overline{\mathrm{T}_{1} / \mathrm{T}_{0}}$ & $\overline{\mathrm{T}_{0}}$ & $\mathrm{~T}_{1}$ & $\mathrm{~T}_{1} / \mathrm{T}_{0}$ \\
\hline Norias & 2.65 & 2.11 & $0.80 *$ & 0.34 & 0.36 & 1.06 & 3.17 & 3.28 & 1.03 & 1.78 & 1.61 & $0.90 *$ \\
\hline El Recreo & 3.67 & 2.12 & $0.58 *$ & 0.23 & 0.42 & $1.83^{*}$ & 3.42 & 3.27 & 0.96 & 1.88 & 1.64 & $0.87 *$ \\
\hline El Cinco & 3.19 & 1.99 & $0.62 *$ & 0.22 & 0.37 & $1.68 *$ & 3.67 & 3.03 & $0.82 *$ & 1.70 & 1.91 & $1.12 *$ \\
\hline Palmas Altas & 2.87 & 2.65 & 0.92 & 0.18 & 0.29 & $1.61 *$ & 3.29 & 3.36 & 1.02 & 1.70 & 1.61 & 0.95 \\
\hline Santa Elena & 3.20 & 2.03 & $0.63 *$ & 0.36 & 0.55 & $1.52 *$ & 3.11 & 3.09 & 0.99 & 1.90 & 1.68 & $0.88 *$ \\
\hline El Orégano & 3.28 & 2.61 & $0.79 *$ & 0.42 & 0.34 & $0.81 *$ & 3.13 & 3.37 & $1.08^{*}$ & 1.97 & 1.72 & $0.87 *$ \\
\hline Carbonerillas & 3.68 & 2.86 & $0.78 *$ & 0.31 & 0.34 & 1.10 & 3.55 & 2.97 & $0.84 *$ & 1.66 & 1.59 & 0.96 \\
\hline Matehualilla & 2.78 & 1.63 & $0.59 *$ & 0.18 & 0.52 & $2.89 *$ & 2.93 & 2.64 & $0.90 *$ & 2.22 & 2.06 & 0.93 \\
\hline Ejido Núñez & 2.55 & 1.79 & $0.70 *$ & 0.42 & 0.41 & 0.98 & 2.93 & 2.98 & 1.02 & 1.95 & 1.87 & 0.96 \\
\hline Maguey Verde & 2.15 & 1.96 & 0.91 & 0.20 & 0.40 & $2.00 *$ & 2.89 & 3.19 & $1.10^{*}$ & 2.27 & 1.96 & $0.86^{*}$ \\
\hline El Arenalito & 2.24 & 1.98 & $0.88 *$ & 0.38 & 0.22 & $0.58 *$ & 3.83 & 3.85 & 1.01 & 1.83 & 1.65 & $0.90 *$ \\
\hline San Cristóbal & 2.19 & 1.60 & $0.73 *$ & 0.37 & 0.36 & 0.97 & 3.34 & 3.52 & 1.05 & 2.09 & 1.83 & $0.88 *$ \\
\hline
\end{tabular}

* Diferencias significativas entre $\mathrm{T}_{0} \mathrm{y} \mathrm{T}_{1}(\mathrm{P} \leq 0.05)$.

Además, en las dos regiones geográficas se encontró una amplia variación entre procedencias o poblaciones en la respuesta al estrés por temperatura, lo que indica plasticidad fenotípica en las plantas para ajustarse a este factor ambiental. En la región norte la población de El Recreo, Coah., presentó el mayor incremento relativo en diámetro, pero también un menor crecimiento en altura y en la relación parte aérea-raíz (Cuadro 6), mientras que la población de El Orégano, Zac. aumentó la asignación de recursos a la raíz pero no al crecimiento en diámetro del tallo. La población de El Cinco, Coah. redujo en casi 20 $\%$ la asignación de recursos a la raíz, lo que ocasionó un aumento en la relación parte aérea-raíz y una fuerte reducción en el incremento en altura. En la región sur sólo las procedencias de Matehualilla y Maguey Verde aumentaron el diámetro del tallo, y esta última población presentó además un aumento en la asignación de recursos a la raíz. En la procedencia de El Arenalito, en cambio, se redujo en $42 \%$ el engrosamiento del tallo, respuesta que contrastó con el resto de las procedencias (Cuadro 6).

\section{Variación en la respuesta de las procedencias al estrés hídrico}

En todas las características evaluadas se encontró interacción $(\mathrm{P}<0.05)$ entre los tratamientos de humedad y las procedencias (Cuadro 2). Al parecer, en $P$. pinceana existe una amplia variación entre procedencias en el crecimiento de las plantas y en su capacidad de adaptación al estrés hídrico, como se ha reportado en Gliricidia sepium, especie leñosa con una amplia distribución en climas cálidos y secos (García-Figueroa y VargasHernández, 2000). Las poblaciones de $P$. pinceana del norte redujeron en mayor grado la acumulación de biomasa del tallo, pero produjeron mayor número de ramas que las del sur, en respuesta al déficit hídrico (Cuadro 7); bajo condiciones de sequía, las poblaciones del norte acumularon menos biomasa que las del sur, debido principalmente a una menor asignación de recursos al tallo.

Dentro de cada región geográfica se observó una amplia variabilidad entre las poblaciones en su respuesta al estrés hídrico. En la región norte, las procedencias de Norias y El Orégano presentaron las mayores reducciones en la relación parte aérea-raíz, pero con menor reducción en la acumulación de biomasa; en el caso de Norias, esta respuesta se logró a expensas de reducir el crecimiento en altura y en diámetro del tallo (Cuadro 7); en cambio, las procedencias de Santa Elena, Coah. y Carbonerillas, Zac. tuvieron menores reducciones en la relación parte aérearaíz y en el crecimiento en altura, pero fueron las más afectadas en la acumulación de materia seca total (Cuadro 7). En la población de El Arenalito, Hgo. ubicada en la región sur, a pesar de tener una de las relaciones parte aérea-raíz más bajas en condiciones de sequía, fue muy afectada en su crecimiento en altura y en acumulación de biomasa; esta es una de las poblaciones con mayor potencial de crecimiento en condiciones favorables de humedad, pero muy sensible al estrés hídrico. En cambio, la procedencia de Ejido Núñez también del sur, redujo en mayor grado la relación parte aérea-raíz y la menor reducción en la acumulación de biomasa, en comparación con las demás poblaciones de esta región (Cuadro 7). 
Cuadro 7. Repuesta relativa al estrés por sequía $\left(S_{1} / \mathbf{S}_{0}\right)$ de las procedencias de Pinus pinceana en las diferentes características de crecimiento de las plantas.

\begin{tabular}{|c|c|c|c|c|c|c|c|c|}
\hline \multirow{2}{*}{$\begin{array}{l}\text { Procedencia } \\
\text { (población ) }\end{array}$} & \multicolumn{3}{|c|}{ Incremento } & \multicolumn{4}{|c|}{ Biomasa } & \multirow{2}{*}{$\begin{array}{l}\text { Relación } \\
\text { parte } \\
\text { aérea/raíz }\end{array}$} \\
\hline & Altura & Diámetro & $\begin{array}{c}\text { Número de } \\
\text { ramas }\end{array}$ & Tallo & Hojas & Raíz & Total & \\
\hline Norias & $0.12 *$ & $0.46^{*}$ & $0.51^{*}$ & $0.66^{*}$ & $0.80 *$ & 0.95 & $0.82 *$ & $0.79 *$ \\
\hline El Recreo & $0.24 *$ & $0.58^{*}$ & $0.21 *$ & $0.60 *$ & $0.74 *$ & $0.82 *$ & $0.73 *$ & $0.85^{*}$ \\
\hline El Cinco & $0.20 *$ & $0.57 *$ & $0.65^{*}$ & $0.80 *$ & $0.75^{*}$ & $0.81 *$ & $0.78^{*}$ & 0.94 \\
\hline Palmas Altas & $0.16^{*}$ & $0.60 *$ & $0.34 *$ & $0.56^{*}$ & $0.67 *$ & $0.77 *$ & $0.68 *$ & $0.82 *$ \\
\hline Santa Elena & $0.24 *$ & $0.59 *$ & $0.47 *$ & $0.65^{*}$ & $0.67 *$ & $0.71 *$ & $0.68 *$ & $0.91 *$ \\
\hline El Orégano & $0.22 *$ & $0.46^{*}$ & $0.37 *$ & $0.73 *$ & $0.83^{*}$ & 0.97 & $0.85^{*}$ & $0.81 *$ \\
\hline Carbonerillas & $0.24 *$ & $0.57 *$ & $0.55^{*}$ & $0.73 *$ & $0.78^{*}$ & $0.81 *$ & $0.78^{*}$ & 0.95 \\
\hline Matehualilla & $0.28 *$ & $0.60 *$ & $0.27 *$ & $0.73 *$ & $0.74 *$ & $0.83^{*}$ & $0.76^{*}$ & $0.85^{*}$ \\
\hline Ejido Núñez & $0.19 *$ & $0.40^{*}$ & $0.43 *$ & $0.83 *$ & $0.89 *$ & 1.02 & 0.92 & $0.81 *$ \\
\hline Maguey Verde & $0.18^{*}$ & $0.54 *$ & $0.32 *$ & $0.68^{*}$ & $0.71 *$ & 0.90 & $0.76^{*}$ & $0.76^{*}$ \\
\hline El Arenalito & $0.11^{*}$ & $0.44 *$ & $0.60 *$ & $0.69 *$ & $0.75^{*}$ & $0.81^{*}$ & $0.75^{*}$ & 0.92 \\
\hline San Cristóbal & $0.13 *$ & $0.53 *$ & $0.15^{*}$ & $0.77 *$ & $0.85^{*}$ & 0.94 & $0.86^{*}$ & $0.86^{*}$ \\
\hline
\end{tabular}

*Representa diferencias significativas entre $\mathrm{S}_{0} \mathrm{y} \mathrm{S}_{1}(\mathrm{P} \leq 0.05)$.

No se encontró una correlación significativa entre el potencial de crecimiento de las poblaciones en condiciones favorables y su respuesta al estrés hídrico, pero sí con la respuesta al estrés por temperatura, en donde hubo una correlación positiva con la acumulación de biomasa del follaje y total. En cambio, la relación parte aérea-raíz en condiciones favorables se asoció en forma negativa con la acumulación de biomasa en la raíz en sequía, y en forma positiva con la relación parte aérea-raíz en las dos condiciones separadas de estrés. Esto indica que las procedencias utilizaron diferentes mecanismos de ajuste en la producción y distribución de biomasa ante las dos condiciones de estrés, y que la relación parte aérea-raíz es mejor indicador de la respuesta de las procedencias a los dos tipos de estrés que el potencial de crecimiento de las plantas. Es evidente que existe plasticidad fenotípica en estas poblaciones, con estrategias diferentes para adaptarse a las condiciones de estrés hídrico y térmico en su ambiente natural.

Córdoba et al. (2008) no encontraron diferencias significativas entre poblaciones de $P$. pinceana en la relación parte aérea-raíz, una de las variables importantes en la respuesta al estrés detectada en este estudio. Sin embargo, en el estudio de Córdoba et al. (2008) se utilizaron plántulas de tres meses de edad que crecían en dos tipos de suelo, y es probable que a esa edad y en esas condiciones no se manifestaran las diferencias entre poblaciones.

\section{CONCLUSIONES}

Los resultados obtenidos demuestran que existe una amplia variabilidad en el potencial de crecimiento y en la respuesta de $P$. pinceana a condiciones de estrés hídrico y por altas temperaturas entre sitios de procedencia geográfica. La especie muestra diversidad genética en las características de crecimiento relacionadas con la acumulación y asignación de biomasa, situación que favorece su capacidad de adaptación al gradiente ambiental que existe dentro de su área de distribución natural en las zonas áridas de la República Mexicana. Aunque la magnitud del efecto ocasionado por los dos tipos de estrés sobre las plantas difirió entre sí y entre procedencias, en ambos casos se detectó que la relación parte aérea-raíz es una característica importante en la respuesta adaptativa de las plantas.

Las poblaciones de Norias, El Orégano y Ejido Núñez, que asignan más recursos a la raíz, presentaron un mejor desempeño en condiciones de estrés hídrico y de temperatura elevada, por lo que sería conveniente utilizar estas poblaciones con fines de restauración ecológica y de conservación de la especie. Además, estos resultados permiten suponer que la especie tendría posibilidades de sobrevivir y adaptarse en las primeras fases de cambio climático que se anticipan, ya que es capaz de ajustar la asignación de biomasa y el engrosamiento del tallo en función de las condiciones de estrés a las que se enfrenta, ya sea por sequía o por altas temperaturas. Sin embargo, con los datos de este estudio no es posible pronosticar el comportamiento de la especie en sitios donde los cambios esperados en temperatura promedio rebasen $\operatorname{los} 2{ }^{\circ} \mathrm{C}$.

\section{AGRADECIMIENTOS}

A SEMARNAT-CONACYT por el financiamiento recibido a través del proyecto 2002-C01-1429 "Ecología, genética de poblaciones y estrategias de conservación de poblaciones naturales de Pinus pinceana". Al M.C. Celestino Flores López por su apoyo en el trabajo de campo y colecta de la semilla en el norte de México. 


\section{BIBLIOGRAFÍA}

Cantin D, M F Tremblay, M J Lechowicz, C Potvin (1997) Effects of $\mathrm{CO}_{2}$ enrichment, elevated temperature, and nitrogen availability on the growth and gas exchange of different families of Jack pine seedlings. Can. J. For. Res. 27:510-520.

Córdoba R D, J J Vargas H, J A Arévalo R (2008) Adaptabilidad de poblaciones de Pinus pinceana en dos suelos. Rev. Fitotec. Mex. 31:273-279.

Cregg B M (1994) Carbon allocation, gas exchange and needle morphology of Pinus ponderosa genotypes known to differ in growth and survival under imposed drought. Tree Physiol. 14:883-898.

García-Figueroa M, J J Vargas-Hernández (2000) Growth and biomass allocation of Gliricidia sepium seed sources under drought conditions. J. Sustain. For. 10:45-50.

Hernández P C, J J Vargas H, C Ramírez H, A Muñoz O (2001) Variación geográfica en la respuesta a la sequía en plántulas de Pinus greggii Engelm. Ciencia For. Méx. 26:61-79.

Kolb P F, R Robberecht (1996) High temperature and drought stress effects on survival of Pinus ponderosa seedlings. Tree Physiol. 16:665-672.

Kozlowski T T (1979) Tree Growth and Environmental Stresses. University of Washington Press, Seattle, WA. 194 p.

Ledig F T, M A Capó A, P D Hodgskiss, H Sbay, C Flores L, M Thompson C, B Bermejo V (2001) Genetic diversity and the mating system of a rare mexican piñon, Pinus pinceana, and a comparison with Pinus maximartinezii (Pinaceae). Amer. J. Bot. 88:1977-1987.

Martínez T T, J J Vargas H, J López U, A Muñoz O (2002) Respuesta al déficit hídrico en Pinus leiophylla: acumulación de biomasa, desarrollo de hojas secundarias y mortandad de plántulas. Terra 20:291-301.

McDaniel R G (1982) The physiology of temperature effects on plants. In: Breeding Plants for Less Favorable Environments. M N Christiansen, Ch F Lewis (eds). John Wiley \& Sons. USA. pp:13-45.
McMillin J D, M R Wagner (1995) Effects of water stress on biomass partitioning of Ponderosa pine seedlings during primary root growth and shoot growth periods. For. Sci. 41:594-610.

Molina-Freaner F, P Delgado, D Piñero, N Pérez-Nasser, E AlvarezBuylla (2001) Do rare pines need different conservation strategies? Evidence from three Mexican species. Can. J. Bot. 79:131-138.

Ngugi M R, M A Hunt, D Doley, P Ryan, P Dart (2003) Dry matter production and allocation in Eucalyptus cloeziana and Eucalyptus argophloia seedlings in response to soil water deficits. New For. 26:187-200.

Perry J P (1991) The Pines of Mexico and Central America. Timber Press, Portland, Oregon, USA. 231 p.

Ramírez-Herrera C, T Beardmore, J Loo (2008) Overcoming dormancy of Pinus Pinceana seeds. Seed Sci. Technol. 36:120.

Samuelson L J, J R Seiler (1993) Interactive role of elevated $\mathrm{CO}_{2}$, nutrient limitations, and water stress in the growth responses of red spruce seedlings. For. Sci. 39:348-358.

SAS Institute (1988) SAS/STAT Users's; release 6.03. SAS Institute, Cary, N.C. 1028 p.

Schlesinger W H, J Lichter (2001) Limited carbon storage in soil and litter of experimental forest plots under increased atmospheric $\mathrm{CO}_{2}$. Nature 411:466-469

Susiluoto S, F Berninger (2007) Interactions between morphological and physiological drought responses in Eucalyptus microtheca. Silva Fenn. 41:221-229.

Teskey R O, R E Will (1999) Acclimation of loblolly pine (Pinus taeda) seedlings to high temperatures. Tree Physiol. 19:519525 .

Zha T, A Ryyppo, K Wang, S Kellomaki (2001) Effects of elevated carbon dioxide concentration and temperature on needle growth, respiration and carbohydrate status in field-grown Scots pine during the needle expansion period. Tree Physiol. 21:1279-1287.

Zhang J, J D Marshall, L Fins (1996) Correlated population differences in dry matter accumulation, allocation, and wateruse efficiency in three sympatric conifer species. For. Sci. 42:242-249. 